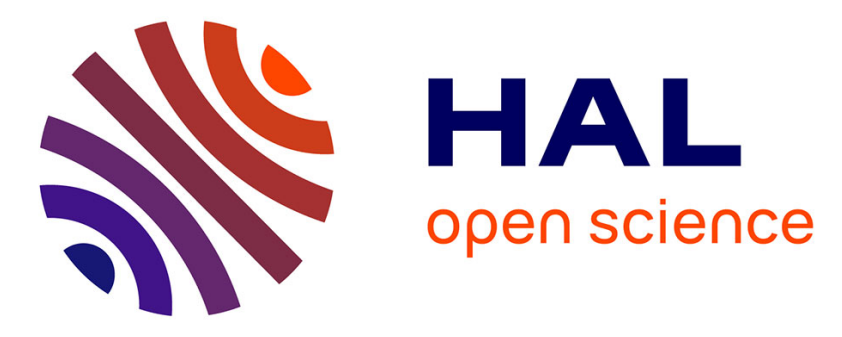

\title{
Telling Stories about Dynamic Networks with Graph Comics
}

\author{
Benjamin Bach, Natalie Kerracher, Kyle Wm. Hall, Sheelagh Carpendale, \\ Jessie Kennedy, Nathalie Henry Riche
}

\section{To cite this version:}

Benjamin Bach, Natalie Kerracher, Kyle Wm. Hall, Sheelagh Carpendale, Jessie Kennedy, et al.. Telling Stories about Dynamic Networks with Graph Comics. Proceedings of the Conference on Human Factors in Information Systems (CHI), ACM, New York, United States, May 2016, New York, United States. 10.1145/2858036.2858387 . hal-01256099

\section{HAL Id: hal-01256099 \\ https://hal.inria.fr/hal-01256099}

Submitted on 14 Jan 2016

HAL is a multi-disciplinary open access archive for the deposit and dissemination of scientific research documents, whether they are published or not. The documents may come from teaching and research institutions in France or abroad, or from public or private research centers.
L'archive ouverte pluridisciplinaire HAL, est destinée au dépôt et à la diffusion de documents scientifiques de niveau recherche, publiés ou non, émanant des établissements d'enseignement et de recherche français ou étrangers, des laboratoires publics ou privés. 


\section{Telling Stories about Dynamic Networks with Graph Comics}

\author{
Benjamin Bach \\ Microsoft Research-Inria Joint \\ Centre, Saclay, France \\ benj.bach@gmail.com
}

\author{
Sheelagh Carpendale \\ Univesity of Calgary \\ Calgary, AB \\ sheelagh@ucalgary.ca
}

\author{
Natalie Kerracher \\ Edinburgh Napier University \\ Edinburgh, UK \\ N.Kerracher@napier.ac.uk
}

\author{
Jessie Kennedy \\ Edinburgh Napier University \\ Edinburgh, UK \\ J.Kennedy@napier.ac.uk
}

\author{
Kyle Wm. Hall \\ Univesity of Calgary \\ Calgary, AB \\ hallkw@ucalgary.ca
}
Nathalie Henry Riche
Microsoft Research
Redmond, WA
nath@microsoft.com

\begin{abstract}
In this paper, we explore graph comics as a medium to communicate changes in dynamic networks. While previous research has focused on visualizing dynamic networks for data exploration, we want to see if we can take advantage of the visual expressiveness and familiarity of comics to present and explain temporal changes in networks to an audience. To understand the potential of comics as a storytelling medium, we first created a variety of comics during a 3 month structured design process, involving domain experts from public education and neuroscience. This process led to the definition of 8 design factors for creating graph comics and propose design solutions for each. Results from a qualitative study suggest that a general audience is quickly able understand complex temporal changes through graph comics, provided with minimal textual annotations and no training.
\end{abstract}

\section{Author Keywords}

Information Visualization, Data-Driven Storytelling, Network Visualization, Dynamic Networks, Comics

\section{ACM Classification Keywords}

H.5.m. Information Interfaces and Presentation (e.g. HCI): Miscellaneous

\section{INTRODUCTION}

Networks are used in sociology, biology, economics and many other domains to model phenomena and obtain insights. While the visualization community has produced a large number of techniques for data exploration, research on how to communicate insights gained from previous explorations is much sparser. Currently, storytelling draws upon different visualization techniques [41]. However, while matrix representations, for example, proved effective to explore dense or dynamic networks, node-link diagrams are much

Permission to make digital or hard copies of all or part of this work for personal or classroom use is granted without fee provided that copies are not made or distributed for profit or commercial advantage and that copies bear this notice and the full citation on the first page. Copyrights for components of this work owned by others than ACM must be honored. Abstracting with credit is permitted. To copy otherwise, or republish, to post on servers or to redistribute to lists, requires prior specific permission and/or a fee. Request permissions from permissions@ acm.org.

CHI'16, May 07 - 12, 2016, San Jose, CA, USA.

Copyright is hold by the owner/author(s). Publication rights licensed to ACM. ACM 978-1-4503-3362-7/16/05 \$15.00.

http://dx.doi.org/10.1145/2858036.2858387

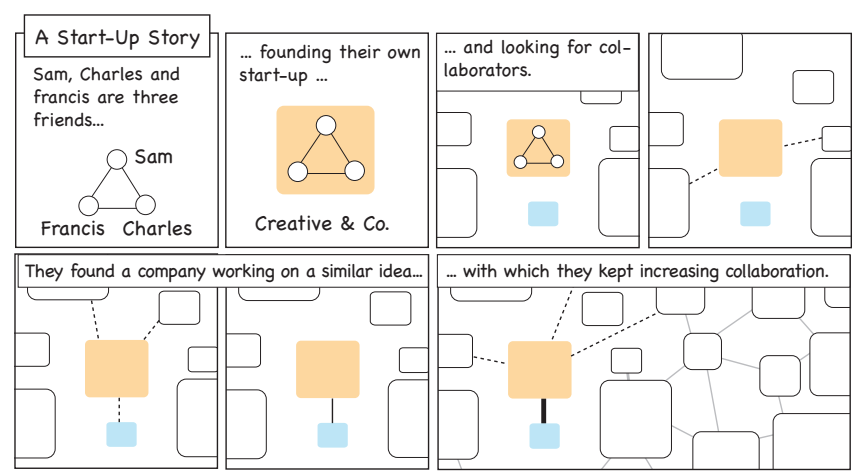

Figure 1. Graph comic: Evolving fictitious business relations.

more familiar to a wide audience and require almost no training, hence are thought of as being preferable for communication. Recent research investigated how to communicate static networks [43]. However, facilitating the communication of temporal changes in dynamic networks-inherently more complex - remains an open challenge.

In this paper, we explore a communication strategy inspired by comics: a popular communication medium. Comics combine visuals and text into an expressive and flexible language [35] to convey sequences of events. These capabilites are promising for communicating temporal changes in complex structures such as networks. Advantages of comics include: being usable in printed and digital format; supporting both textual and oral descriptions; as well as standing by themselves by tightly integrating narration with visuals.

Our work takes a first look at how graph comics (Figure 1) can convey changes occurring in dynamic networks. While McCloud [35, 36] presents a comprehensive theory for creating comics in art, we investigate their potential for data-driven storytelling. We report on our iterative process for designing graph comics and present a set of eight factors to consider for creating consistent comics. We present empirical evidence from a qualitative study with 10 participants demonstrating that a general audience can interpret graph comics without training and with minimal textual annotations. Examples of graph comics along with supplementary material from our design process and readability study are available at http://graphcomics .azurewebsites.net. 


\section{RELATED WORK}

There is a considerable literature in visualization for network exploration [21, 47, 17, 19] but almost none on how to communicate insights gained from exploration to an audience. Our work is a first step in this direction.

\section{Visually Exploring Dynamic Networks}

For dynamic networks, research focused on creating interactive visualizations. Three surveys [8, 29, 4] review over a hundred techniques, broadly categorizing them into visualizations based on animations [26, 6], on small multiples [45] where time is mapped to space, and hybrids $[39,5]$.

The focus of this work is on the communication of insights gained from dynamic networks, where structure and/or data attributes carried by vertices and edges vary over time. While none of the existing systems has been explicitly designed for communication, several of them contain strategies to improve the communication of temporal changes in networks. For example, GraphDiaries [6] use animated staged transitions to emphasize sets of nodes added or removed from the network while preserving the overall context. DiffAni [39] uses a combination of small multiples, animation and difference maps to emphasize specific changes at different levels of detail or to convey the overall state of the network. Ideas specifically about how to convey insights about dynamic networks are still missing. For example, how can changes, occurring intermittently over a long period of time, or happening in parallel with many others, be communicated? We directly investigate how techniques from comics can be used to communicate insights about dynamic networks.

\section{Storytelling with Visualizations}

Communicating insights with data visualizations or datadriven storytelling is a topic of increasing interest in the research community [16, 41, 22, 34, 24, 31, 10]. Examples of data-driven storytelling are now commonly used in journalism (e.g., New York Times, Le Monde), and are also successfully employed for scientific communication [34]. Segel and Heer [41] first drew the landscape of storytelling techniques, identifying seven genres. Since then, several authoring systems have appeared. For example SketchStory [32] proposed a technique for live presentation, and Ellipsis [40], Contextifier [23], and sense.us [20] offer an authoring environment to augment existing visualization with annotations and mechanisms to induce a storyline. While recent studies have investigated several storytelling mediums, such as infographics [7, 9] or data videos [2], many of these genres, including comics, have not been studied in relation to their communicative potential for conveying insights about dynamic networks. The most closely related work is Spritzer et al., who aim to support the authoring of communicative node-link diagrams [43], yet without addressing the temporal aspect of networks.

\section{Storytelling with Sequential Art}

Using a sequence of images, akin to small multiples, to communicate changes has been practiced for centuries [4]. These image sequences can be found in a variety of textbooks to illustrate biological or physical processes, in user manuals to convey instructions, and in magazines and newspapers to

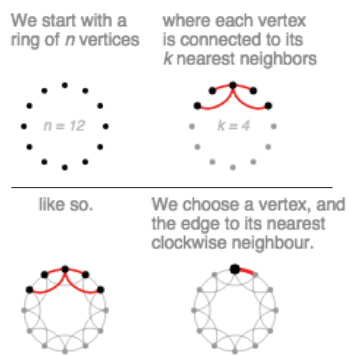

(a)

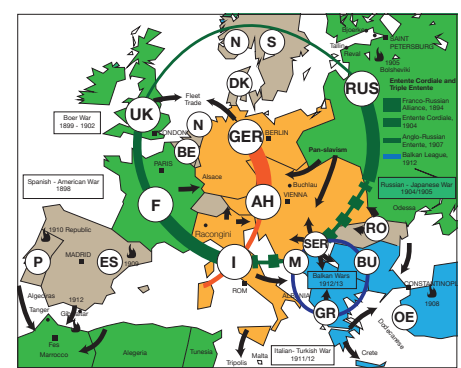

(b)
Figure 2. Diagrams akin to comics: (a) graph generation algorithms by Bret Victor [46], (b) european alliances before WWI (after [15] ).

show weather evolution. Examples of sequential representations to encode network changes exist in network science (Figure 2(a)), and in historical atlases (Figure 2(b)). Read in linear order, sequences of images imply two aspects: progress in depicted time (change), and progress in narrative time (story). In this work, we focus on comics for a variety of reasons. First, this sequential art provides a rich visual language [12] tightly integrating narration with visuals and enabling the illustration of complex changes via the use of symbolic representations and annotations. Comics are also a wellresearched communication medium, thoroughly described by Scott McCloud [35, 36] and Neil Cohn [14] amongst others. This allows us to build upon existing grammars and theories.

Comics have recently inspired information comics [12, 27], which seek to convey concepts, inform, and educate readers through use of the comic medium. They have been employed in areas including education and academia [25], health education [37], and scientific communication [44]. There are not many systems supporting the creation of such comics. Zhao et al. explored ways to manually augment infographics with text and layout panels [49], while others explored an automatic generation of comics from temporal image sequences (including video and photo albums) for storytelling purposes [13], and the assisted creation of textures in traditional comics [28]. Considering storytelling for dynamic networks, comics have the advantage of allowing readers to control the pace at which information is conveyed [13], not relying on the reader's memory of previous events [16] as required by animations or videos. Also, they are able to communicate complex information efficiently and effectively in a small space, in comparison to written text alone [18] in print or digital display alike.

\section{GRAPH COMIC EXAMPLE}

Figure 3 presents a graph comic example to illustrate our discussion on design factors, and is based on Figure 2(b). The example arose from a discussion with a high-school teacher who commented that the configurations of European alliances preceding WWI were consistently difficult to communicate to students. Historical events led to a series of intricate alliances and conflicts evolving over time. Figure 2(b) conveys the temporality of events, emphasizes strategic moves and critical contracts, shows how small events triggered larger events, and which nations were the driving forces, revealing the challenges faced when explaining the evolution of this network. 
European Alliances before World War I (1836-1914)
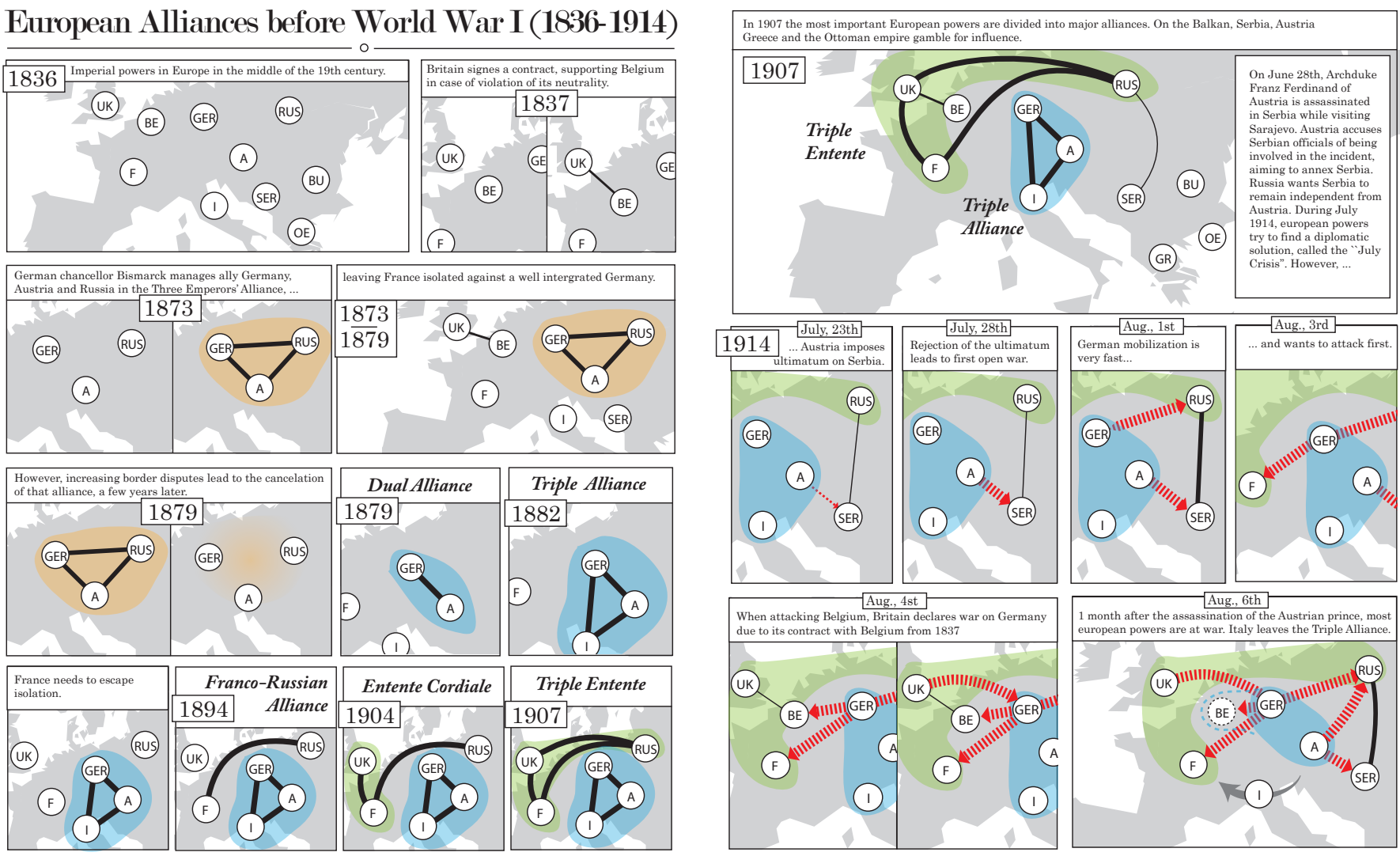

Figure 3. Graph comic example illustrating historical events preceeding WWI, targeted to a general audience.

Our example (Figure 3) breaks down the complexity into logical chunks and provides explanations for each chunk. Text presents information about the events that led to an alliance or to conflicts, while visuals convey the graph topology and changes. Countries and their relationships are depicted using a node-link diagram, laid out over a geographical map. Link width encodes the strength of relation (thick=alliance, thin=support), link color and texture its nature (black=support, red dashed=conflict). Formal alliances are indicated by different colors. The meaning is deduced from the juxtaposition of the visuals and the annotations.

Since events and changes do not happen at a regular pace, showing one panel every year does not highlight key changes. Instead, panels in graph comics represent periods of time, depending on the amount and importance of events and changes. Topological change in graph comics is mainly perceived through differences between consecutive panels (panel 1873). However, to emphasize a particular change, one can use symbols such as arrows or dashed lines (last panel, Aug. 6th). One may also vary the level of detail and direct a reader's attention. For example, several panels present a zoomed-in version of the network (e.g., panel 1837) to direct the reader's attention to a subset of countries that play an important role at this time. In contrast, other panels depict the entire network (e.g., panel 1907) and serve as context, to emphasize an important network topology and summarize previous changes.
Initial feedback from the high-school teacher suggests that graph comics can facilitate communication of temporal changes in networks. Current textbook illustrations such as Figure 2(b), are often used as a reference and summary after attending a lecture and/or reading a text depicting network changes. In contrast, graph comics can support the communication of the changes themselves. After looking at this graph comic, she pointed us towards more example stories in the history curriculum: economic dependencies, collaborations and coalitions between political parties, lineage of royal families, structure of organizations, and influence networks of historical figures or chains of implications.

\section{DESIGN PROCESS}

Our graph comic design process involved 6 steps and many sketches and design charrettes amongst the authors.

\section{Step 1: Understanding Traditional Comics}

We first collected diagrams, comic literature [35, 36], and pictures akin to comics. Since the design space for comics is extremely large, we focused on 5 significant elements in the visual language of traditional comics (Figure 4.1). Panels (Figure 4.1a) are individual pictures showing a moment [35]. This can be a specific time, or a scene without any temporal meaning, e.g., a close-up of a previous panel. A sequence of panels (strip) can convey different states and imply temporal evolution. Readers understand the temporal order of events through order of panels and reading direction conventions, e.g. leftright and top-down in western culture. Finding the right num- 
ber of panels is a key to creating a compelling story that provides sufficient detail but is not weighed down by unimportant ones. Characters are actors in the comic and its story (Figure 4.1b). Characters act and interact in a world possibly including a variety of objects. The world provides the context and stage for a story (Figure 4.1c). We call elements that specifically refer to time and illustrate changes to characters or objects effects. Representations for effects include arrows, speed lines, and textual annotations denoting a sound (Figure 4.1d). Finally, we group narration, speech, thought bubbles, and other textual annotations under text (Figure 4.1e).

Traditional comics use our knowledge of the real world to help interpret their content. As graphs are abstract constructs, we cannot reliably leverage a real-world understanding. We identify five specific challenges for data-driven comics:

C1. Element identity - Characters in traditional comics are recognizable via their distinct visual features. In graph visualization, nodes and edges are usually represented by circles, sometimes using labels for identifications. Variations in shape and color reflect attributes in the data.

C2. Depicting change - In comics, readers can infer changes even if not shown explicitly. For example, a baby being shown implies it has been born. In graphs, a (new) node being shown does not mean it was not there before.

C3. Order of events-Many changes exhibit a natural order (e.g. humans grow old, objects fall down, day turns into night). Changes in graphs do not have a natural order nor duration; a cluster can grow or shrink, divide or merge.

C4. Spatial context-In comics, the spatial context of a scene is usually indicated by the panel background: the same background means the same place and often the same scene. Switching spatial context from one cluster to another may not be recognized by a reader (both consists of nodes and links). C5. Number of elements-Comics usually show a small set of characters in every panel. Changes in graphs, may involve many more elements than it is possible to show in a panel.

\section{Step 2: Designing for Graph Changes}

Our second step was to find possible visual encodings that could represent graph objects, their properties, and the possible changes which they may undergo (Figure 4.2). We derived a list of objects and events based on several taxonomies reporting on graphs and changes in graphs $[33,3,42,48,1$, 30]. Possible changes include node and edge appearance and disappearance, birth of cluster, splitting of clusters. Authors independently sketched possible encodings and reflected on the resulting set in a collaborative session.

Nodes were consistently represented as circles and edges as lines. Clusters were represented in a variety of ways: explicit node-link representations, blobs, or rectangles with sharp or round corners. Possibilities for representing changes were much more diverse, but could be divided into two general categories: those explicitly depicting before and after states (and sometimes a transition state) in separate images (e.g., a cluster growing in size: $\square \square$ ) and those using a single symbolic representation (e.g., cluster growing 恬). Another observation was that arrows are a highly overloaded encoding, being used to represent everything from an edge's addition $\mathrm{O} \rightarrow \mathrm{O}$, as-
1) Traditional Comics

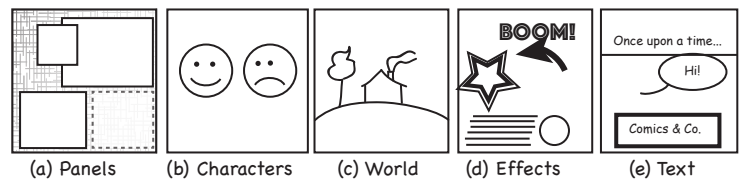

2) Designing for Graph Changes

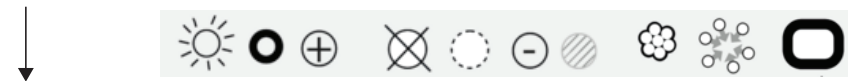

3) Design Principles

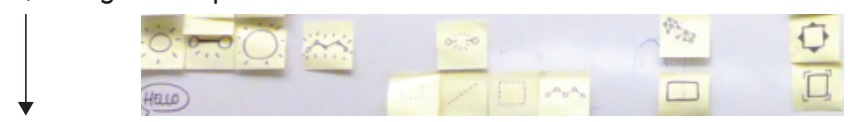

4) Creating Comics

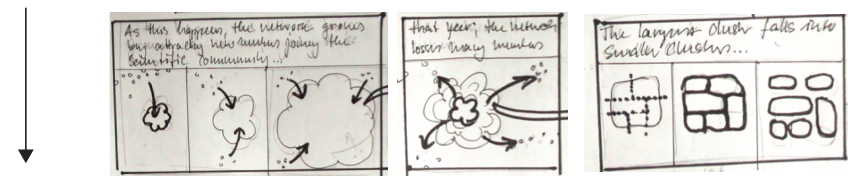

5) Discussion with Domain Experts

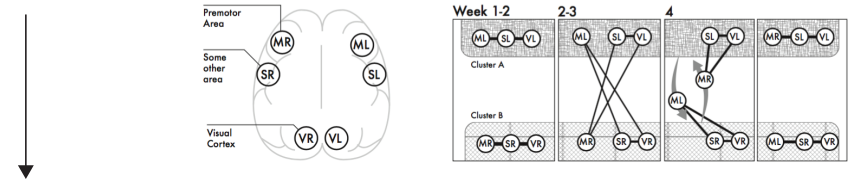

6) Readability Study (see Section "Readability Study")

Figure 4: Design process and intermediate results.

sembly of elements (form, merge, join) or disassembly (e.g., a node leaving a cluster $\square+0$ ), growth, contraction, or continuation in the network's size or density, repetition of a temporal pattern, or to indicate the transition between before and after states. This diverse set called for identifying factors to consider to make conscious and justifiable design decisions and motivated design principles to ensure consistency.

\section{Step 3: Design Principles}

Our design principles define when certain visual marks and their attributes (e.g., circles, line style, arrows) should be used and when not. A visual encoding should not have two different meanings in the same comic; if arrows indicate movement, it should not also encode object removal.

We defined three principles to maintain consistency in our comics: (1) Representation of graph objects (nodes, clusters, paths etc.) must be consistent across types of changes (e.g. if a cluster is initial represented as a square, then it should be consistently represented by a square, regardless of whether it is forming, merging, or dissolving); (2) Similar changes should use similar encodings (e.g. the encoding used to show deletion of nodes should be similar to that used for deletion of edges); (3) Opposite changes should use paired, visually opposite encodings (e.g. an arrow pointing towards a cluster for addition and one pointing away for deletion).

\section{Step 4: Creating Comics}

Using our design challenges and the structural principles, five of the authors sketched about 15 comics for stories found in the scientific literature (social sciences, chemistry) as well as 
the evolution of the authors' research groups. Comics were sketched with pen and paper to have the highest degree of creative freedom (Figure 4.4). Several comics have been created for the same story. We discussed the resulting comics and reflected on the application of the encodings from Step2, as well as aspects beyond simple encoding of change: how to convey parallel events, how to distinguish between actual graph elements and abstractions, how to structure the page. After discussion and several iterations, we converged towards the set of 8 design factors, detailed in Section "Design Factors". Those design factors capture the essence of our design solutions and our considerations when designing graph comics. Some design factors directly address the challenges in creating graph comics (C1-C5), mentioned in Step 1.

\section{Step 5: Discussion with Domain Experts}

To collect external feedback, usage scenarios, and understand if our design factors could guide the creation of real-world graph comics, we contacted two domain experts : a neuroscientist and a high school history teacher. With the neuroscientist we spent several hours spread over one week in close collaboration and discussed two main usage scenarios for graph comics: i) to illustrate brain connectivity changes and study findings in textbooks and in research papers, ii) to sketch hypotheses in grant proposals where no or partial data is available (Figure 4.5). Following our discussions, we sketched according to our design factors, vectorized, and discussed several comics. With the teacher, we had two 1-hour sessions, resulting in Figure 3. During these sketching sessions, we mostly validated our core set of design factors, only minimally altering them to add or modify design solutions.

\section{Step 6: Reading Study}

Finally, to check if graphics comics are readable by a wider audience, we designed a qualitative study, detailed in Section "Reading Study" (Figure 4.6). Three comics were selected and their stories refined, covering as many cases as possible from our design factors. From our study findings, we finalized the description of our design factors and removed design solutions that were not easily understood.

\section{DESIGN FACTORS FOR GRAPH COMICS}

This section presents our resulting 8 design factors for creating graph comics and illustrates our design solutions.

\section{F1: Visual Representation of Graph Elements}

To show characters and objects, most traditional comics use depictions of the real world (C1). The elements we consider in graph comics are more abstract and, for graph comics, limited to three classes: nodes, links, and groups (or clusters). Since node-link diagrams are familiar to a wide audience, we propose representing nodes by circles or points, links by lines, and groups by contours or enclosing shapes (Figure 5). These marks can be further differentiated by using unique shapes, colors, or other visual encodings (Figure 5) as well as textual labels. Throughout a comic, the visual encoding of elements may vary according to their importance with respect to the story (F5) or the current level of detail (F6).
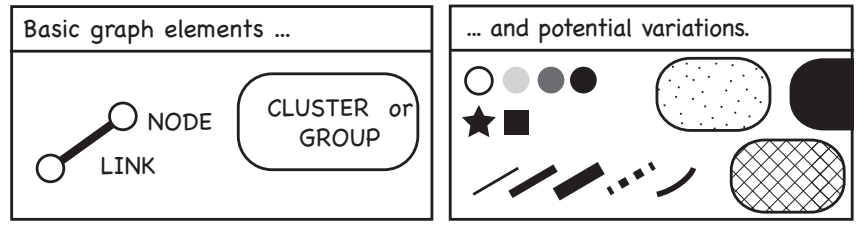

Figure 5: Visual Representation of graph elements.

\section{F2: Visual Representation of Changes}

Panels in comics represent key moments of time and actions or events that constitute the story [36]. In traditional comics, spatial layout and sequencing of panels defines the storyline and its temporality. For graph comics $(\mathrm{C} 2, \mathrm{C} 3)$, we propose to convey temporal change in four distinct ways (Figure 6).

- Before/after states - This technique is the most akin to traditional comics. It does not require representation of the change itself, but rather shows the network (or any subnetwork) at two moments in time before and after the change (Figure 6a). The reader deduces which changes occurred by comparing the content of the two panels, through a mechanism known as closure [35].

- Transitions and fast forwards - Representing complex changes may require more panels, while remaining perceived as a transition, rather than individual states. We propose either reducing the horizontal width of the panels (Figure 6b) or grouping them together in a single panel, separated by thin lines only (Figure 6c).

- Symbolic representation of change - This technique explicitly represents the change using a symbolic representation, which can be added to the current state of the network (Figure 6c). Change can be shown using one of many symbols including arrows to indicate direction of movement, highlighting or sparks to show appearance, or symbols of growth. More examples can be found online.

- Representation of abstract metrics - When the change to be represented is not suited to a compact symbolic representation, alternative techniques can be employed. Figure $6 \mathrm{~d}$ uses a line chart to show changes in cluster density over a certain period. Specific metrics, such as cluster size for a given point in time, can be shown directly numerical labels.

\section{F3: Temporality of Changes}

Telling a story through comics includes selecting important key moments from the data $(\mathrm{C} 2, \mathrm{C} 3)$. Readers of traditional comics may reasonably guess the duration of events by the number of panels used to represent an action and by analogy to the real world (e.g., one panel depicts nighttime, the next depicts daytime). Graph comics require additional techniques and annotations to illustrate changes that occur in parallel or repeat, and to explicitly encode temporal duration.

- Sequential changes - As in traditional comics, we leverage the horizontal order of panels to imply the temporal sequence of the panels, i.e. left to right implies moving forward in time. More panels can imply a longer time period (Figure 7) unless otherwise annotated, and can be used to show the length of a transition (F2). Note that in traditional comics, panel size is not always proportional to duration. Similarly, in graph comics, panels should comfortably fit all necessary elements. 

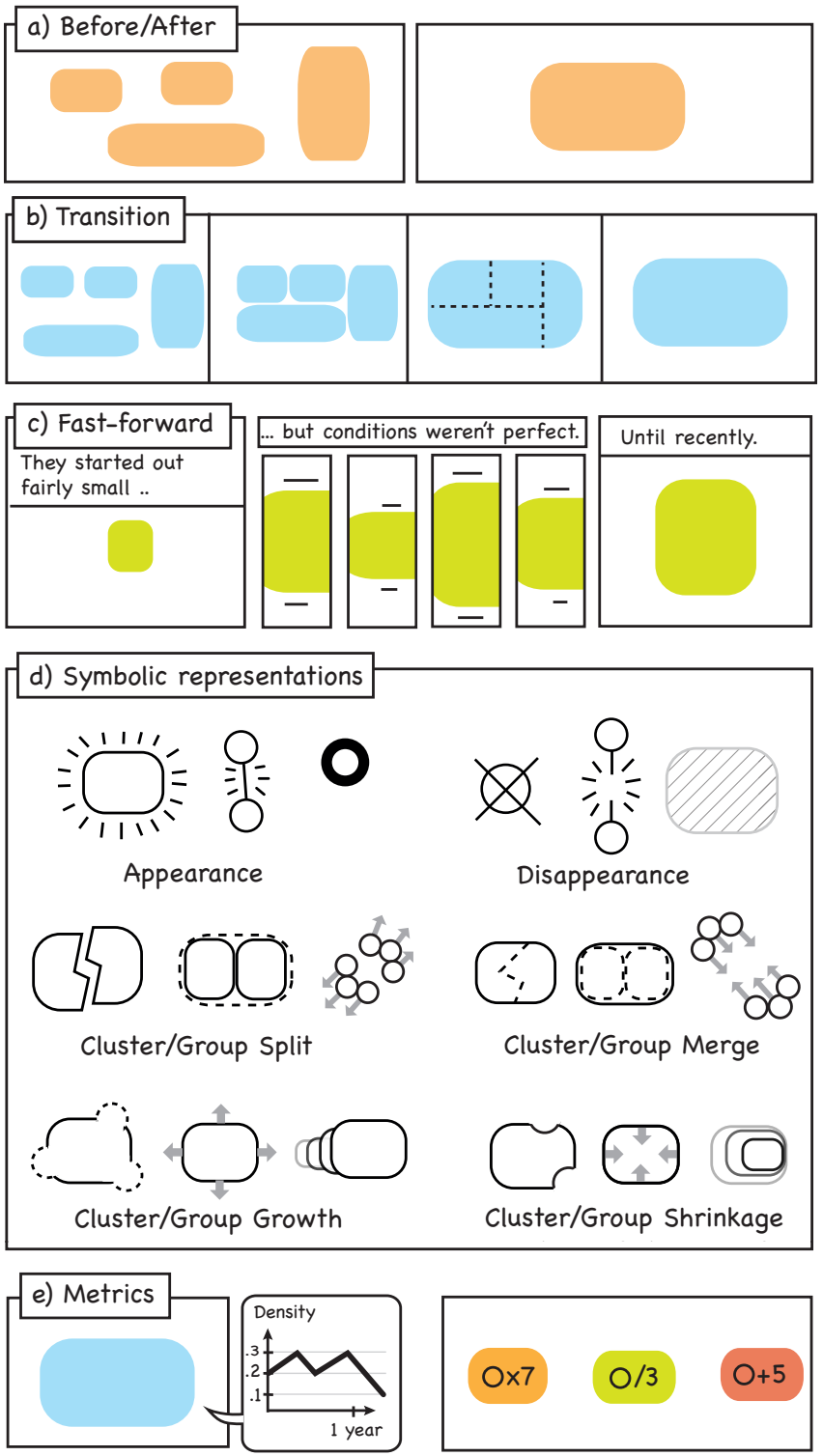

Figure 6: Visual representation of change.

- Parallel changes - Changes happening in parallel are better depicted in the same panel. If not possible, an option is to split the panel vertically by a fine line (Figure 7a) but requires textual annotations to ensure correct interpretation.

- Non-linear temporality - Changes that involve cycling or branching can be encoded by the spatial layout of panels, augmented with arrows indicating reading direction (Figure 7b). Alternatively, symbols, e.g., musical notations encoding repetition, could provide a compact way of representing non-linear temporality but require annotation.

- Symbolic and explicit representation of time - To encode specific temporality of changes, time can be communicated symbolically (e.g., symbols of seasons) or explicitly (Figure 7c). In both cases, we propose mimicking traditional comics and placing this temporal information on boxes above panels or on their top-left corners.
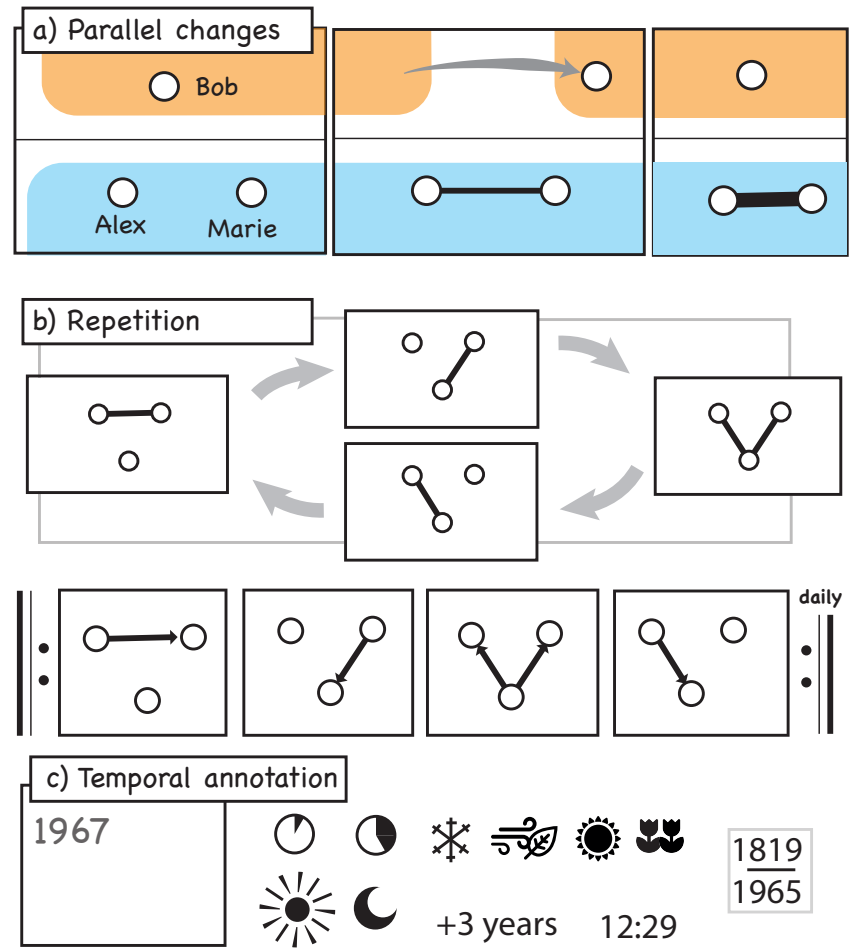

Figure 7: Temporality of change.

\section{F4: Element Identity}

In traditional comics, identification of recurring elements occurs through the identifiable features of a character's physical appearance, and through the character's position within a scene (C1). In graph comics, we propose four alternatives for identifying recurring objects (Figure 8).

- Visual encodings or labels - Mimicking traditional comics, one could use specific visual encodings to differentiate elements (shape or glyphs, color or texture). Labelling of elements often only needs be employed at an element's first occurrence. Note that color has the most impact on people's interpretation.However this strategy may lead to overwhelming visuals as more elements need to be tracked, so should be used in important cases, e.g., a main character. If the visual encoding changes, labels should be repeated.

- Stable position - Another strategy mimicking traditional comics is to preserve the relative position of elements to each other from panel to panel. This can drastically reduce the visual complexity of panels as there can be a large initial panel which contains all labels and all subsequent ones do not contain further labels. However, producing stable layouts for dynamic networks is known to be a challenging task [11]. At least, changes in positions should be clearly identified by a transition or symbolic depiction (F2).

- Polyptych - A strategy to enable readers to track groups across multiple panels is to use a polyptych, i.e. a scene that is assembled across multiple pictures. Polyptychs can help to reduce visual complexity and visually connect related panels (e.g., orange group in Figure 8). 


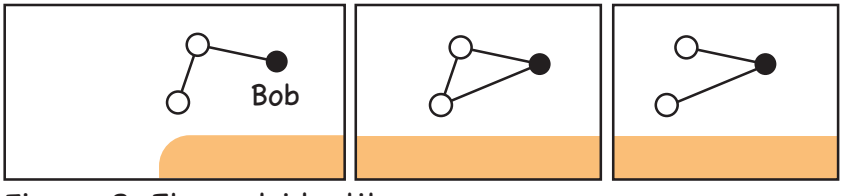

Figure 8: Element identity

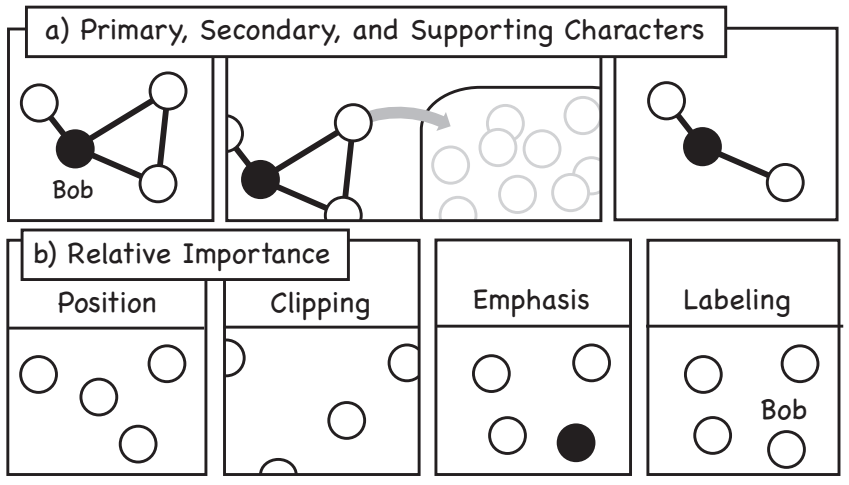

Figure 9: Cast of characters

\section{F5: Cast of Characters}

As stories convey specific insights about the data, they often focus on a subset of characters: main, supporting, and extra characters. The main character(s) is usually one (or a few) elements, on which the story focuses (e.g., the story of Bob in Figure 9a). Supporting characters are related to the main character, e.g., by connection, or are otherwise important for the understanding of the story and are countable (Figure 9b). Extra characters are characters that represent other elements in the graph whose identity and precise number is not of importance (C5). What matters is their existence, nature, and approximate cardinality (Figure 9c). We propose three strategies to distinguish between these types of characters.

- Visual emphasis-Main characters should be visually highlighted using filled node shapes, labelling, or color if more than one main character is present. For example, Figure 9 shows the main character as a filled circle, while supplementary characters are outlined. Extra characters are visually deemphasized.

- Position-Elements in the center of the panel imply more importance than elements on the margin. A specific case of positioning is clipping, where a graph element is only partially visible. Clipped elements are less important than non-clipped elements, though still play a role in the current panel. Also, several clipped elements imply several more hidden ones.

- Labelling-Main characters need only be marked by labels the first time they appear providing that their identity is preserved thereafter $(\mathrm{F} 4)$.

\section{F6: Level of Detail}

Related to the previous consideration is the level of detail in describing changes. Figure 10 illustrates an example of addition of nodes to a group (a) and the connections between two groups (b) at four levels of details.
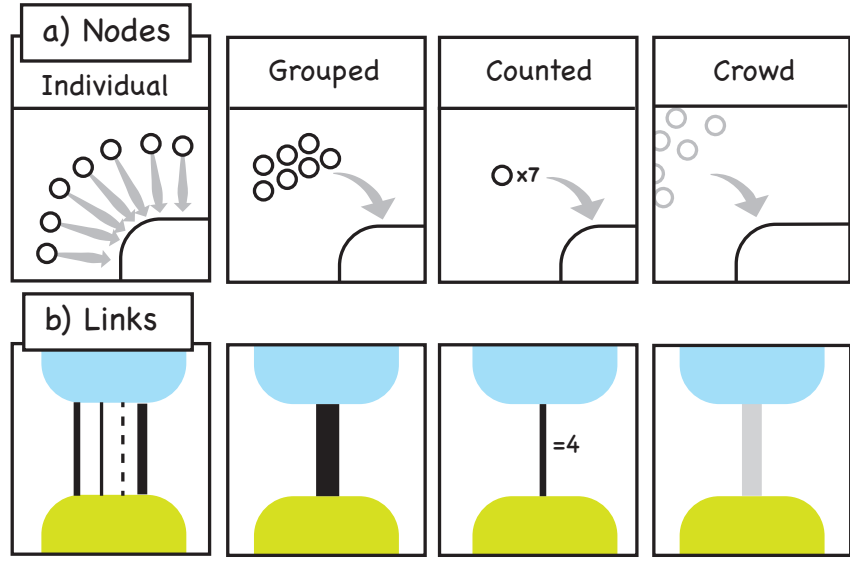

Figure 10: Level of detail

- Individual-Elements are depicted individually, and every element has its own visual effect (movement in this case).

- Grouped-Elements are depicted individually, but same effects are grouped.

- Counted - A single element representing the group is depicted, annotated by a number to indicate cardinality.

- Crowd-Elements are shown deemphasized and less organized (F5) to imply they are not countable. Their goal is to simply express existence.

\section{F7: Overview and Detail}

Often the focus of a story switches from a larger collection of elements, e.g., an entire network or a set of groups, to fewer elements, e.g., nodes in a group (C4). Graph comics offer several ways to show transitions between overview and detail.

- Bleed-Figure 11a shows the entire network on the left, outside of any panel. This technique called bleed is used in comics to set the scene for the following panels [35]. A bleed figure can be a picture of the entire network in comic style or a screenshot from an actual visualization, or any other graphic that is necessary (timeline, geographical or anatomical maps, etc.). Figure 11a further shows two different stories, related to the same network, using color to mark character identity. While bleed can be used to set the context for the entire story, the following techniques can be used to show transitions through panels.

- Cut-out-In a cut-out (Figure 11b), a part of the graph is highlighted (e.g., a dashed line), to which the following panels refer. The same works in the opposite direction.

- In-place-While a cut-out focuses attention entirely on a subgraph, if the larger context is important, details can be shown in-place, similar to a fisheye lens (Figure 11c).

- Zoom-In a zoom transition (Figure 11d), the part of the network is gradually enlarged, mimicking a camera zoom in movies. This transition may be the most akin to traditional comics and leaves more space for annotations.

\section{F8: Representation of Multivariate Networks}

There are cases where it is convenient to encode additional information in the visual appearance of graph elements, such 


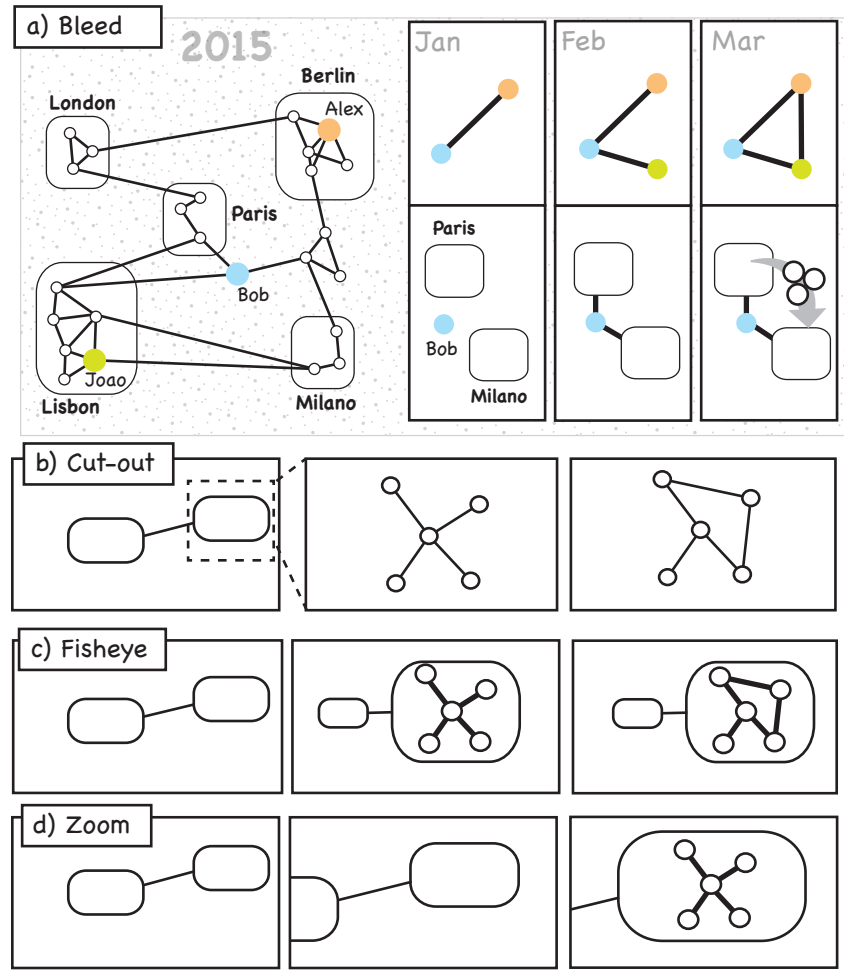

Figure 11: Overview and detail

as node type (e.g., male, female), edge type (e.g., married, friend), edge weight or group density. It is possible to map those characteristics to visual variables (Figure 12). For example, employing size for node degree, line width for edge strength, color (hue) to differentiate different node or edge types, and lightness to indicate density in groups. To avoid visual complexity, visual variables must be applied with care. There are three major considerations:

- Ambiguity-Color usage should be limited, if possible to a maximum two purposes. For example, indicating node types or maintaining identity of nodes (F4). If color is necessary for node types, node identity could be maintained by location or repeated labelling.

- Legend- Except if using a visual convention, we generally recommend providing a legend or textual annotations (Figure 12) as they are difficult to deduce.

- Fidelity - Several visual variables (i.e. size, line strength, lightness) are continuous and creating an exact mapping of the underlying values is unlikely to be effective. For example, if conveying relative differences between node degree can be achieved with node size (Figure 12 (left)), conveying a decrease of degree of two in successive panel is unlikely to succeed. While we suggest being as accurate as possible when mapping data dimensions to visual variables, if an exact interpretation is required, we suggest explicitly annotating visuals with text (Figure 12(right)).

\section{READING STUDY}

To investigate if a wider audience could read and interpret graph comics, we conducted a qualitative study, asking 10 participants from diverse backgrounds to interpret graph comics. Our hypotheses were the following:
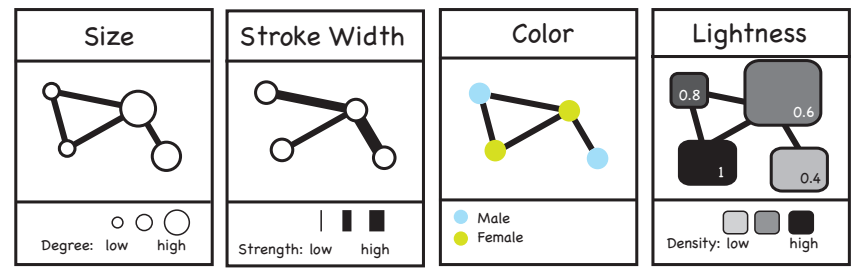

Figure 12: Representation of multivariate networks

H1 - graph comics can be understood without training, i.e. readers can correctly interpret the story, and,

H2 - ambiguities can be resolved via discussion.

Findings from our study were used to improve our set of design factors. The designs in this paper are the final ones. Study material is available online.s

\section{Study Setup}

Methodology - We followed an iterative methodology akin to rapid iterative testing (RITE [38]) to investigate the readability of graph comics. We prepared three initial comics including visuals and annotations. After each session, we iterated on the designs that were ambiguous to participants, and steadily decreased the amount of annotations to assess how interpretable the visuals were without text. Figure 3 shows a comic with complete visuals and annotations, while Figure 13 shows a comic with all text stripped, as given to our last participants.

Participants-Participants were recruited by an independent service and told that their gratuity was independent of their performance. Rather than deciding a priori on a number of participants, our criteria was to gather enough evidence that comics could be understood by non-experts (the case after 10 participants and 2 pilots). None of our participants was knowledgable in graph analysis or visualization. The age average was 34.1 years. Two participants were female.

Material-We prepared three comics: 1) the history of a start-up company and its business relationships (Start-up), 2) a family and its changing friends (Family), and 3) a criminal network (Criminal, Figure 13). Initially, we had text added that explains the context of change, but not the changes themselves to investigate if people could decode them accurately (e.g., panel 4, Figure 13: "The police start taking action"). Each comic contained 16 or 18 individual panels, comfortably fitting one page. Each panel contained between one and three findings we wanted participants to report on (H1), e.g., two sub-groups join green group and 1 sub-group joints lime group (Figure 139)

Procedure - To ensure participants would vocalize their interpretations, we ran sessions in pairs. We held five 1-hour sessions under the same conditions. The experimenter explained social network terminology: nodes, links, and groups (which contain nodes) using a simple sheet. The experimenter did not describe any comic components or visual encodings. Each participant received a color copy of the same comic and was encouraged to first read and annotate them before discussing among themselves to reach a consensus. To avoid distracting them, the experimenter left the study 


\begin{tabular}{l|l|r|r|r|r|r|r} 
Comic & Findings & 1 & 2 & 3 & 4 & 5 & Avg. \\
\hline \hline Start-up & 14 & N/A & 3.3 & 5.1 & 1.4 & 2.4 & $\mathbf{3 . 1}$ \\
Family & 21 & 9.2 & 8.5 & 12.2 & 13.0 & 4.4 & $\mathbf{1 4 . 1}$ \\
Criminals & 21 & 6.5 & 8.4 & 7.3 & 7 & 4.1 & $\mathbf{8 . 1}$ \\
\hline
\end{tabular}

Table 1. Time (sec.) it took participant pairs to discuss each comic.

room and observe in a separate room. Video and audio was recorded at all times. We provided comics in the same order for all pairs: Start-up, Family, Criminals. Discussions for each comic was split into two parts. The first part involved only the two participants discussing the comics. In the second part, the experimenter came back in the room and asked one of the participants to tell the story that they had agreed upon. Participants reported their interpretations and the experimenter asked for clarification and findings participants did not report explicitly if necessary.

Analysis-We created a table that listis findings for each panel and coded the videos according to $a$ ) which findings participants verbalized (table found online) and $b$ ) how long it took each pair to verbalize them (Table 1). In addition, after each pair we collected a list of ambiguous encodings and possibly adjusted the comic.

\section{Study Findings}

All participants generally correctly interpreted all three comics, independently of the amount of annotations present (H1). While annotations undoubtedly helped participants resolve ambiguities and interpret comics faster, these were not essential for understanding comics (H2).

Quantitative findings. 43 findings (76\%) were verbalized by all 5 pairs, with 53 findings (94\%) found by 4 pairs. The Start-up comic was entirely discussed in 3.1 minutes on average. This discussion involved the time it took participants to read through the comic, understand the changes, formulate those changes in the context of the story, and discuss ambiguities with their peer. The Family and Criminal comics were entirely discussed in 14.1 and 8.1 minutes, respectively.

Change is understood in context. The specific story context (criminals, family, start-up) has a strong impact on the interpretation of graph changes. For example, several participants associated groups in the criminal networks with different geographical areas, and consequently inferred that arrows meant a physical move in addition to joining a cluster (i.e., joining a different section of the city, P3:"they are spatially divided"). In cases where a particular change could not be identified from a single panel or a short sequence, participants interpreted them by relating it to i) the story context (P4 about (6): "the spy [then] removed the godfather") or ii) by looking at preceding and succeeding panels. For example, our inconsistent use of dotted lines for different graph events (link forming, group disappearance, group split) was decoded accurately with before/after panels.

Symbolic representations require annotations. While symbolic representations and conventions generally require learning, we hypothesized that encodings restricted to depicting graph changes were discoverable without any annotations or training. As participants puzzled over the initial symbols we used, we iterated over different sets. For example, we iter-

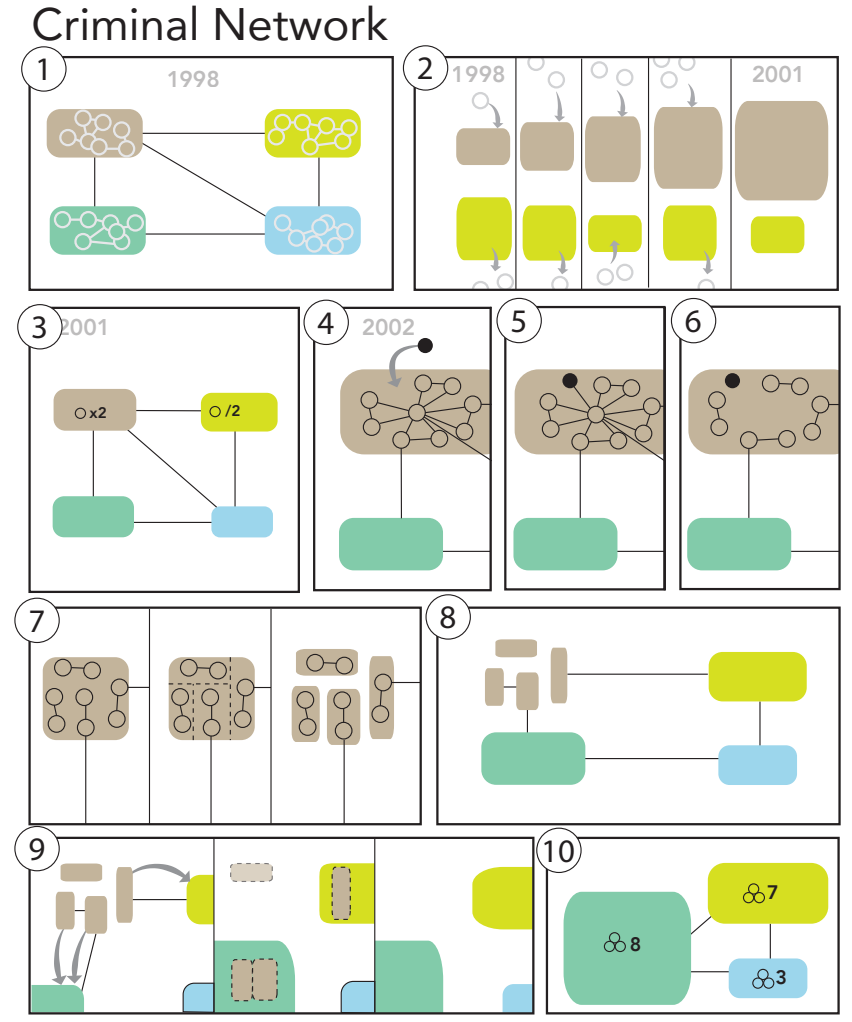

Figure 13. Example story as used in our reading study. Circled numbers references panels for our discussion.

ated over node, edge, and group disappearance using dashed lines, then reduced opacity of dashed lines, then crossed out elements. The semantics of the cross was more consistently understood as deletion, while others were not. The use of arrows (Figure 13 (2)(4)(9), indicating movement of a node or group, was also discovered easily. However, any other symbolic encodings without annotations were ambiguous and discussed in the context of preceding and succeeding panels. We conclude that symbolic representations generally require annotation or explanation when they first occur.

Insights on our 8 design considerations. Visual encodings borrowed from node-link diagrams are appropriate (F1) and participants understood before/after states, transitions and metrics (F2) to encode graph changes. The number of panels (F3) was perceived as proportional to the duration of a change. Thus, while complex changes may require more panels to be understandable (e.g. Figure 1 (7)), they may be considered longer lasting if not annotated (P2 about (9): "[...] in the process of disapearing but is not already gone."). Parallelity of panels (via horizontal panel split, inclined panel split or nested panels) was not perceived at all without annotations.

Relative positions, size, and color are important to maintain element identity across panels (F4), though color receives priority in making decisions: in one case where a graph element was colored differently in a succeeding frame, P6 replied that "I assume if it were the same, the color would be the same". Clipped, overlapping and somewhat varied graphical elements (Figure 13 (1)) were correctly interpreted as the existence of objects rather than interpreted as specific number and 
identity (sF5). Considering the levels of detail (F6), link line thickness was consistently interpreted as strength of the relation (Figure 13 (10)-(13)) and dashed lines and contours perceived as weak or uncertain by most participants (P2: "met the people but are not actually friends"). Participants interpreted zooming (F7) (Figure 13 (4)-(6)) without difficulty. Finally, the visual encoding of attributes (F8) requires an additional hint or a legend. For example, group size was not consistently interpreted as cardinality (a monetary profit in the case of the criminal network). Adding nodes entering the group (Figure 13 (2)) clarified this encoding.

\section{Study limitations}

Our results suggest that participants were able to understand the story in our comics either directly (H1) or could clarify the meaning in discussion $(\mathrm{H} 2)$. Our results do not mean that our designs, e.g. crossing-out elements to indicate removal, are the best to use, but that they are understandable and mostly unambiguous. "Good designs" depend on many factors and alternative designs could be evaluated in the future. A limitation of our current study is the low number of participants and the iterative refinement of the comics during the study, therefore our quantitative findings should be taken with care. Our goal was to refine and iterate over our design factors and we stopped at 10 participants as we did not find novel issues or ambiguities that may have caused adaptions. Further studies, more controlled and with potentially more participants, can help quantify which visual encodings are easier to understand and investigate more deeply how people decode comics, e.g., if they get an overview first, or read them panel-by-panel.

\section{DISCUSSION AND FUTURE WORK}

In this work, we described initial investigations into using comics to communicate changes in networks. We report below conclusions to our set of research questions.

\section{Can we create comics to convey changes in networks?}

Graph comics emerged from a series of discussions amongst the authors of this paper and two domain scientists, interweaving reviews of existing comics grammars and dynamic network task taxonomies; explorations of a design space for representing graph changes symbolically; and practical sketching sessions of dynamic network stories. Through this iterative process, we generated a wealth of graph comics, involving a wide range of sequencing strategies, visuals and textual annotations. It became clear that comics are a flexible and expressive medium, enabling communication with a variety of visuals and text. Our initial research question evolved into whether it was possible to create graph comics in a more systematic manner and without relying too much on text. To answer this question, we refined a set of key design factors and used these as guidelines to generate graph comics more systematically. While there are still a large number of possibilities to convey the same dynamic network story, we offer these design considerations to provide guidance for generating consistent graph comics.

\section{Are graph comics a promising storytelling medium?}

Our next question in this work was to assess whether these graph comics could be used for storytelling. We tackled this question from two different angles: can a general audience understand graph comics without any training?, and would people who could potentially use these comics in their communications find them useful? Our reading study with 10 novice participants provided initial evidence that graph comics can be understood by a wider audience, with minimal textual annotations and without any training. Our discussions with and feedback from a high-school teacher and a neuroscientist illustrated that graph comics can be used in a variety of scenarios. Both these findings indicate that graph comics are a promising storytelling medium.

\section{Can we support the authoring of graph comics?}

We identified that we can create graph comics to express changes in networks and that they are readable by a wider audience and applicable to a variety of usage scenarios. A logical following research question is about authoring. Graph comics presented in this paper were created manually, iterating with pen and paper and eventually vectorizing them. This process was laborious enough that it is unclear whether people would create graph comics without any support. While investigating proper authoring support is out of scope of this paper, we discuss several directions for future research.

First, we observed that through this project, we streamlined the creation process for graph comics, suggesting authoring steps in a particular order. Further research on how experts draw comics and how to streamline the authoring process would be essential to extract a set of implications for the design of an editing tool. From our experience, a lightweight editor for graph comics, extending an existing SVG-editor for example, would greatly improve the authoring process. It could consist of an interface that allows creation and layout of panels, creating, placing, and customizing graph elements, and adding textual annotations. Laborious manual tasks can be supported, such as the creation of sets of elements and the duplication and layout of panels, providing visual templates for higher-level changes and transitions, as well as supporting the creation of simple charts and data-to-visual mappings. Considering graph comics as an output of existing network visualization tools raises further challenges. In the case of large graphs, for example, the author could be assisted in extracting important parts of the network (e.g., with respect to a selected node, or set of "main actors") and abstracting the rest (F5, F10). He could also be guided towards a temporal segmentation with salient events and meaningful states [5].

To conclude, we demonstrated the potential of comics to communicate temporal changes in networks. We hope it will motivate further research on the topic, providing foundations for authoring systems, and extending the application of comics to tell stories of other types of data structures.

\section{ACKNOWLEDGMENTS}

We thank our collaborators Tara Madhyastha (Radiology, University of Washington) and Friederike Bach (Soemmerda High School, Germany), and our reviewers. This work was partially being funded by NSERC, the Izaak Walton Killam Pre-Doctoral Scholarship Program, the NSERC Vanier CGS Program, and AITF. The first three authors contributed equally to the discussion and development of the ideas in this paper. 


\section{REFERENCES}

1. Jae-Wook Ahn, Catherine Plaisant, and Ben Shneiderman. 2014. A Task Taxonomy for Network Evolution Analysis. IEEE Transactions on Visualization and Computer Graphics 20, 3 (2014), 365-376.

http://doi.ieeecomputersociety.org/10.1109/ TVCG. 2013.238

2. Fereshteh Amini, Nathalie Henry Riche, Bongshin Lee, Christophe Hurter, and Pourang Irani. 2015. Understanding Data Videos : Looking at Narrative Visualization through the Cinematography Lens. In Proceedings of the ACM Conference on Human Factors in Computing Systems (CHI). ACM, 1459-1468.

3. Sitaram Asur, Srinivasan Parthasarathy, and Duygu Ucar. 2007. An event-based framework for characterizing the evolutionary behavior of interaction graphs. Proceedings of the ACM International Conference on Knowledge Discovery and Data Mining (KDD) (2007), 913. DOI :

http://dx.doi.org/10.1145/1281192.1281290

4. Benjamin Bach, Pierre Dragicevic, Daniel Archambault, Christophe Hurter, and Sheelagh Carpendale. 2016. A Descriptive Framework for Temporal Data Visualizations Based on Generalized Space-Time Cubes. Computer Graphics Forum (2016). to appear.

5. Benjamin Bach, Natalie Henry Riche, Tim Dwyer, Tara Madhyastha, Jean-Daniel Fekete, and Thomas Grabowski. 2015. Small MultiPiles: Piling Time to Explore Temporal Patterns in Dynamic Networks. Computer Graphics Forum 34, 3 (2015), 31-40. DOI : http://dx.doi.org/10.1111/cgf.12615

6. Benjamin Bach, Emmanuel Pietriga, and Jean-Daniel Fekete. 2014. GraphDiaries: Animated Transitions and Temporal Navigation for Dynamic Networks. IEEE Transactions on Visualization and Computer Graphics 20, 5 (2014), 740-754. DOI : http: //dx. doi .org/ 4077CBD5-B2C4-4EC6-9475-E3D0687E96A4

7. Scott Bateman, Regan L. Mandryk, Carl Gutwin, Aaron Genest, David McDine, and Christopher Brooks. 2010. Useful Junk?: The Effects of Visual Embellishment on Comprehension and Memorability of Charts. In Proceedings of the ACM Conference on Human Factors in Computing Systems (CHI). ACM, New York, NY, USA, 2573-2582. DOI :

http://dx.doi.org/10.1145/1753326.1753716

8. Fabian Beck, Michael Burch, Stephan Diehl, and Daniel Weiskopf. 2014. The State of the Art in Visualizing Dynamic Graphs. In Proceedings of the Eurographics Conference on Visualization (EuroVis), State-of-The-Art-Reports.

9. Michelle A. Borkin, Azalea A. Vo, Zoya Bylinskii, Phillip Isola, Shashank Sunkavalli, Aude Oliva, and Hanspeter Pfister. 2013. What Makes a Visualization Memorable? IEEE Transactions on Visualization and Computer Graphics (2013).
10. Jeremy Boy, Jean-Daniel Fekete, and Françoise Détienne. 2015. Storytelling in Information Visualizations: Does it Engage Users to Explore Data?. In Proceedings of the ACM Conference on Human Factors in Computing Systems (CHI), ACM (Ed.). ACM, Seoul, South Korea, 1449-1458. DOI : http://dx.doi.org/10.1145/2702123.2702452

11. Ulrik Brandes and Martin Mader. 2012. A Quantitative Comparison of Stress-Minimization Approaches for Offline Dynamic Graph Drawing. In Graph Drawing, Marc Kreveld and Bettina Speckmann (Eds.). Lecture Notes in Computer Science, Vol. 7034. Springer, 99-110. DOI : http://dx.doi.org/10.1007/978-3-642-25878-7_11

12. Joshua Caldwell. 2012. Information comics: An overview. IEEE International Professional Communication Conference (2012). DOI : http://dx.doi.org/10.1109/IPCC.2012.6408645

13. Wei-Ta Chu, Chia-Hsiang Yu, and Hsin-Han Wang. 2015. Optimized Comics-Based Storytelling for Temporal Image Sequences. Multimedia, IEEE Transactions on 17, 2 (2015), 201-215. DOI : http: //dx.doi.org/10.1109/TMM. 2014.2383616

14. Neil Cohn. 2013. The Visual Language of Comics: Introduction to the Structure and Cognition of Sequential Images. Bloomsburry Academic.

15. dtv Verlagsgesellschaft. 2000. dtv-Atlas Weltgeschichte. Band 2: Von der franzoesischen Revolution bis zur Gegenwart.

16. N Gershon and W Page. 2001. What Storytelling can do for Information Visualization. Communications of the ACM, 44, 8 (2001), 31-37.

17. H. Gibson, J. Faith, and P. Vickers. 2013. A Survey of Two-Dimensional Graph Layout Techniques for Information Visualisation. Information Visualization 12 , 3-4 (2013), 324-357. DOI :

http://dx.doi.org/10.1177/1473871612455749

18. Michael Green and Kimberly Myers. 2010. Graphic Medicine: Use of Comics in Medical Education and Patient Care. British Medical Journal 340, c863 (2010). DOI : http://dx.doi.org/10.1136/bmj.c863

19. Steffen Hadlak, Heidrun Schumann, and Hans-Jörg Schulz. 2015. A Survey of Multi-faceted Graph Visualization. In Eurographics Conference on Visualization (EuroVis), State-of-the-Art-Reports, R Borgo, F Ganovelli, and I Viola (Eds.). The Eurographics Association. DOI :

http://dx.doi.org/10.2312/eurovisstar.20151109

20. Jeffrey Heer, Fernanda B. Viégas, and Martin Wattenberg. 2007. Voyagers and Voyeurs: Supporting Asynchronous Collaborative Information Visualization. In Proceedings of the ACM Conference on Human Factors in Computing Systems (CHI). ACM, New York, NY, USA, 1029-1038. DOI :

http://dx.doi.org/10.1145/1240624.1240781 
21. Ivan Herman, Guy Melancon, and Scott M. Marshall. 2000. Graph Visualization and Navigation in Information Visualization: A Survey. Visualization and Computer Graphics, IEEE Transactions on 6, 1 (2000), 24-43. http://ieeexplore.ieee.org/xpls/abs_all. jsp?arnumber $=841119 \&$ tag $=1$

22. Jessica Hullman and Nick Diakopoulos. 2011. Visualization Rhetoric: Framing Effects in Narrative Visualization. IEEE Transactions on Visualization and Computer Graphics 17, 12 (2011), 2231-2240. DOI : http://dx.doi.org/10.1109/TVCG.2011.255

23. Jessica Hullman, Nicholas Diakopoulos, and Eytan Adar. 2013a. Contextifier: Automatic Generation of Annotated Stock Visualizations. In Proceedings of the ACM Conference on Human Factors in Computing Systems (CHI). ACM, New York, NY, USA, 2707-2716. DOI : http://dx.doi.org/10.1145/2470654.2481374

24. Jessica Hullman, Steven Drucker, Nathalie Henry Riche, Bongshin Lee, Danyel Fisher, and Eytan Adar. 2013b. A Deeper Understanding of Sequence in Narrative Visualization. IEEE Transactions on Visualization and Computer Graphics 19, 12 (2013), 2406-2415. DOI : http://dx.doi.org/10.1109/TVCG.2013.119

25. Aaron Humphrey. 2014. Beyond Graphic Novels: Illustrated Scholarly Discourse and the History of Educational Comics. Media International Australia 73 (2014).

26. Daniel McFarland James Moody and Skye Bender-deMoll. 2005. Dynamic Network Visualization. American Journal of Sociololgy (2005).

27. Heike Elisabeth Jüngst. 2010. Information Comics: Knowledge Transfer in a Popular Format. Lang, Frankfurt. 366 pages.

28. Rubaiat Habib Kazi, Takeo Igarashi, Shengdong Zhao, and Richard DavisNat. 2012. Vignette: Interactive Texture Design and Manipulation with Freeform Gestures for Pen-and-ink Illustration. In Proceedings of the ACM Conference on Human Factors in Computing Systems (CHI). ACM, New York, NY, USA, 1727-1736. DOI : http://dx.doi.org/10.1145/2207676.2208302

29. Natalie Kerracher, Jessie Kennedy, and Kevin Chalmers. 2014. The Design Space of Temporal Graph Visualisation. In Proceedings of the Eurographics Conference on Visualization (EuroVis), Short Papers Track, N. Elmqvist, M. Hlawitschka, and J. Kennedy (Eds.). The Eurographics Assocation, Swansea, 7-11. DOI :

http://dx.doi.org/10.2312/eurovisshort. 20141149

30. N Kerracher, J Kennedy, and K Chalmers. 2015. A Task Taxonomy for Temporal Graph Visualisation. IEEE Transactions on Visualization and Computer Graphics 21, 10 (Oct. 2015), 1160-1172. DOI :

http://dx.doi.org/10.1109/TVCG.2015.2424889
31. Robert Kosara and Jock MacKinlay. 2013. Storytelling: The Next Step for Visualization. IEEE Computer (Special Issue on Cutting-Edge Research in Visualization) 46, 5 (2013), 44-50. DOI : http://dx.doi.org/10.1109/MC.2013.36

32. Bongshin Lee, Rubaiat Habib Kazi, and Greg Smith. 2013. SketchStory: Telling more Engaging Stories with Data Through Freeform Sketching. IEEE Transactions on Visualization and Computer Graphics 19, 12 (2013), 2416-2425. DOI :

http://dx.doi.org/10.1109/TVCG.2013.191

33. Bongshin Lee, Catherine Plaisant, Cynthia Sims Parr, Jean-Daniel Fekete, and Nathalie Henry. 2006. Task Taxonomy for Graph Visualization. In Proceedings of the 2006 AVI workshop on BEyond time and errors: novel evaluation methods for information visualization. ACM, 1-5.

34. Kwan-Liu Ma, I. Liao, J. Frazier, H. Hauser, and H.-N. Kostis. 2012. Scientific Storytelling Using Visualization. IEEE Computer Graphics and Applications 32, 1 (Jan 2012), 12-19. DOI : http://dx.doi.org/10.1109/MCG .2012.24

35. Scott McCloud. 1994. Understanding Comics: The Invisible Art. HarperPerennial, New York.

36. Scott McCloud. 2006. Making Comics: Storytelling Secrets of Comics, Manga and Graphic Novels. Harper, New York.

37. Sarah McNicol. 2014. Humanising Illness: Presenting Health Information in Educational Comics. Medical Humanities 40, 1 (2014), 49-55. DOI : http://dx.doi.org/10.1136/medhum-2013-010469

38. M.C. Medlock, D. Wixon, M. McGee, and D Welsh. 2005. The Rapid Iterative Test and Evaluation Method: Better Products in Less Time. In Cost-Justifying Usability, Second Edition: An Update for the Internet Age, Randolph G. Bias and Deborah J. Mayhew (Eds.). 489-518.

39. Sébastien Rufiange and Michael J McGuffin. 2013. DiffAni: Visualizing Dynamic Graphs With a Hybrid of Difference Maps and Animation. IEEE Transactions on Visualization and Computer Graphics 19, 12 (Dec. 2013), 2556-65. DOI :

http://dx.doi.org/10.1109/TVCG. 2013.149

40. Arvind Satyanarayan and Jeffrey Heer. 2014. Authoring Narrative Visualizations with Ellipsis. Computer Graphics Forum 33, 3 (2014), 361-370. DOI : http://dx.doi.org/10.1111/cgf. 12392

41. Edward Segel and Jeffrey Heer. 2010. Narrative Visualization: Telling Stories with Data. IEEE Transactions on Visualization and Computer Graphics 16, 6 (2010), 1139-48. DOI : http://dx.doi.org/10.1109/TVCG.2010.179

42. Ross Shannon, Aaron Quigley, and Paddy Nixon. 2009. Time sequences. In Proceedings of the ACM Conference 
on Human Factors in Computing Systems, Extended Abstracts (CHI). ACM, 4615-4620.

43. Andre Suslik Spritzer, Jeremy Boy, Pierre Dragicevic, Jean-Daniel Fekete, and Carla Maria Dal Sasso Freitas. 2015. Towards a Smooth Design Process for Static Communicative Node-Link Diagrams. In Computer Graphics Forum, Vol. 34. Wiley-Blackwell, 461-470. DOI : http://dx.doi.org/10.1111/cgf.12658

44. M. Tatalovic. 2009. Science Comics as Tools for Science Education and Communication: A Brief, Exploratory Study. Journal of Science Communication 8, 4 (2009).

45. Edward R. Tufte. 1983. The Visual Display of Quantitative Information. Graphics Press, Cheshire, CT.

46. Bret Victor. 2011. Scientific Communication As Sequential Art. http: //worrydream.com/\#! /ScientificCommunicationAs SequentialArt. (2011). online, last accessed Sept., 24, 2015.

47. T. von Landesberger, A. Kuijper, T. Schreck, J. Kohlhammer, J.J. van Wijk, J.-D. Fekete, and D.W. Fellner. 2011. Visual Analysis of Large Graphs: State-of-the-Art and Future Research Challenges. Computer Graphics Forum 30, 6 (April 2011), 1719-1749. DOI : http: //dx.doi.org/10.1111/j.1467-8659.2011.01898.x

48. Ji Soo Yi, Niklas Elmqvist, and Seungyoon Lee. 2010. TimeMatrix: Analyzing Temporal Social Networks Using Interactive Matrix-Based Visualizations. International Journal of Human-Computer Interaction 26, 11-12 (nov 2010), 1031-1051. DOI :

http://dx.doi.org/10.1080/10447318.2010.516722

49. Z. Zhao, R. Marr, and N. Elmqvist. 2015. Data Comics: Sequential Art for Data-Driven Storytelling. Technical Report. Human Computer Interaction Lab, University of Maryland. 